\title{
Spatial-Resolved EELS Studies on Single-Walled Nanotubes Based on B, C and N
}

\author{
R. Arenal ${ }^{*, * *}$, O. Stephan***, A. Loiseau ${ }^{*}$ \\ * LEM, CNRS-ONERA, 92322 Châtillon, France \\ ${ }^{* *}$ Lab. Microscopias Avanzadas, Inst. Nanociencia Aragon (INA), U. Zaragoza, Zaragoza (Spain) \\ ${ }^{* * *}$ LPS, CNRS-Université Paris Sud, 91405 Orsay, France
}

The effect of incorporate heteroatoms (B, N or BN) into carbon nanotubes' (C-NTs) network could be a perfect way of control their opto-electronic properties [1-4]. As a matter of fact, the band gap of the NTs can be tuned in the visible range and, for pure BN-NTs, even in the UV spectral range. However, it is worth noting that a very detailed analysis of the atomic configuration and concentration of the different species of these heteroatomic nanotubes is highly required in order to determine their impact on the electronic properties of the NTs. In this communication, we present a detailed study of the atomic configuration of different kinds of heteroatomic single-walled (SW) NTs: CNx and BxCyNz via spatial-resolved (SR) EELS. We have examined the different chemical species present in the NTs, determined their average concentration as well as their spatial distribution within the walls and studied their chemical environment and bonding.

These spatial-resolved EELS experiments have been carried out in a dedicated STEM VGHB501 $(100 \mathrm{KV})$. We used a slightly defocused probe of few $\mathrm{nm}$ in diameter with a convergence angle of $15 \mathrm{mrad}$ and collection angle of $24 \mathrm{mrad}$. The energy resolution was close to $0.7-0.8 \mathrm{eV}$. This spectroscopic information was obtained using the spectrum-imaging (SPIM) acquisition mode [5]. Particular attention has been devoted to contamination and damage during acquisition.

Fig. 1(a) displays a TEM image of a CNx-SWNTs sample produced via CVD technique. The tubes are well-crystallized, and very few defects are observed. Local chemical characterization of as-grown nanotubes has been carried out by SR-EELS analyses, revealing a mean nitrogen content of 1.7 atom \%. Fig. 1(b) shows a representative example of the EELS spectrum recorded of those NTs. This EELS spectrum corresponds to the sum of 6 EEL spectra acquired on the bundle of NTs shown in the bright field image. From the C-K edge it can be deduced that the nanotubes consist of a typical graphitic network with $\mathrm{sp}^{2}$-type bonding and that the tubes are very well graphitized $[1,3,4,6]$. The inset of Fig. 1(c) shows the N-K edge. Two features can be observed at $\sim 398$ and $\sim 402 \mathrm{eV}$, respectively. They could be attributed to the $\pi^{*}$ states of the pyridine-like and graphitic-like configuration, respectively.

The case of BxCyNz-SWNTs is even more challenging. As a matter of fact, recently, it has been shown that $\mathrm{SW}-\mathrm{BxCyNz}$ nanotubes are composed of $\mathrm{BN}$ nano-domains (1-2 nm large) embedded into a graphene layer, building then $\mathrm{BN}-\mathrm{C}$ heterojunctions [7]. These nanostructures are assumed to be the result of the phase separation existing at equilibrium in bulk systems between hBN and graphite. Fig. 2 displays a TEM micrograph from a sample produced via arc discharge technique. The analysis of the spectrum-imaging (SPIM) recorded on the region shown in the HAADF image (Fig. 2 (a)) revel the presence of a BN nanodomain of $\sim 1.5 \times 10 \mathrm{~nm}$, as well as BN nanoparticles which can be observed, see B chemical map Fig. 2 (b). Furthermore, all these results will be compared to our results on pure BN-SWNTs [8].

\section{References}

[1] R. Arenal, X. Blase, A. Loiseau, Advances in Physics 59, 101 (2010).

[2] P. Ayala, R. Arenal, A. Loiseau, A. Rubio, T. Pichler, Rev. Mod. Phys. 82, 1843 (2010).

[3] P. Ayala, R. Arenal, M. Rummeli, A. Rubio, T. Pichler, Carbon 48, 575 (2010). 
[4] C.P. Ewels, M. Glerup, J. Nanosci. and Nanotech. 5, 1345 (2005).

[5] C. Jeanguillaume, C. Colliex, Ultramicroscopy 28, 252 (1989).

[6] H. Lin, R. Arenal, S. Enouz-Vedrenne, O. Stephan, A. Loiseau, J. Phys. Chem. C 113 (2009).

[7] S. Enouz-Vedrenne, O. Stephan, A. Loiseau, J. Phys. Chem. C 112, 16422 (2008).

[8] R. Arenal, F. De La Pena, O. Stephan, M. Walls, M. Tence, A. Loiseau, C. Colliex, Ultramicros. (2008).

[9] These works were supported by the European Comm. under the FP6, STREP project BNC Tubes, NMP4-CT-2006-03350 and by the ANR PNANO (project Cedona). CVD CNx-SWNTs samples were produced by T. Susi and E. Kauppinen (U. Helsinki) and arc discharge BxCyNz-SWNTs by N. Arutyunyan and E. Obratzsova (A.M. Prokhorov General Physics Institute Moscow). We thank these teams for having kindly provided these samples and fruitful discussions.
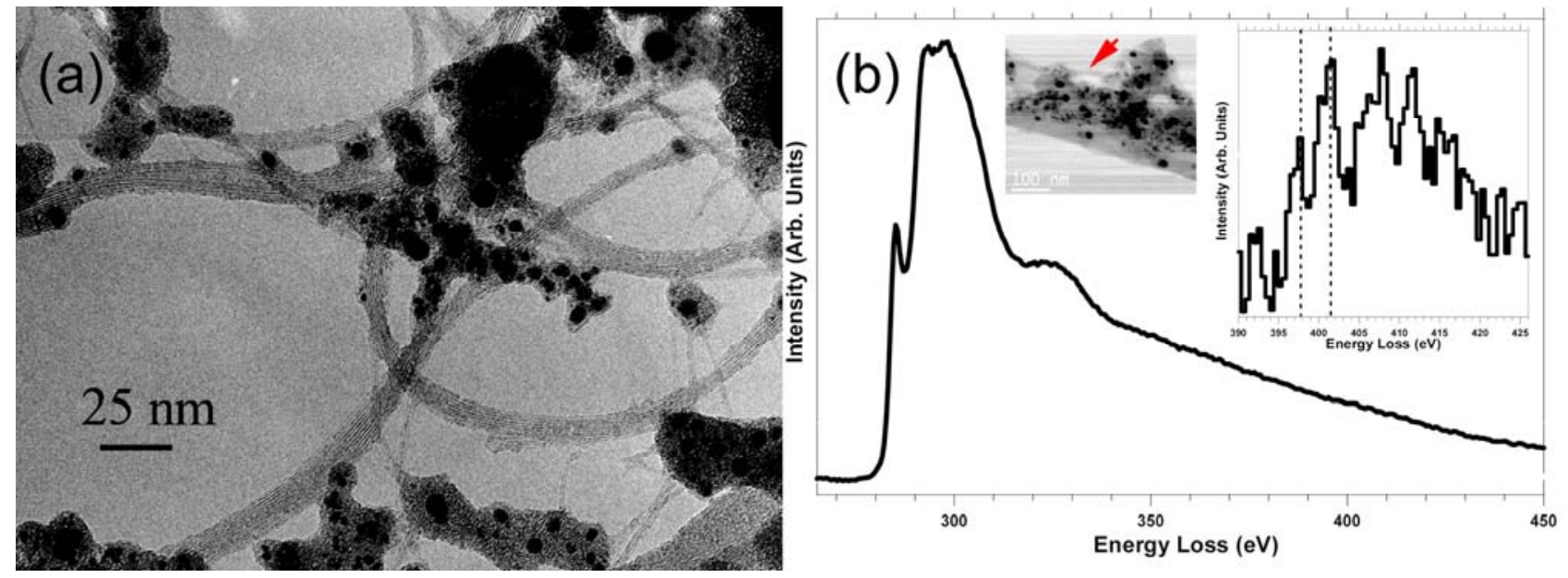

Figure 1. (a) HRTEM image of dispersed CNx-SWNTs, showing a mixture of NTs (bundles and individual) and catalyst nanoparticles. (b) Sum of 6 EEL spectra recorded on the bundle of CNx-SWNTs displayed at the inset (marked with an arrow). Inset: N-K edge obtained after background subtraction.
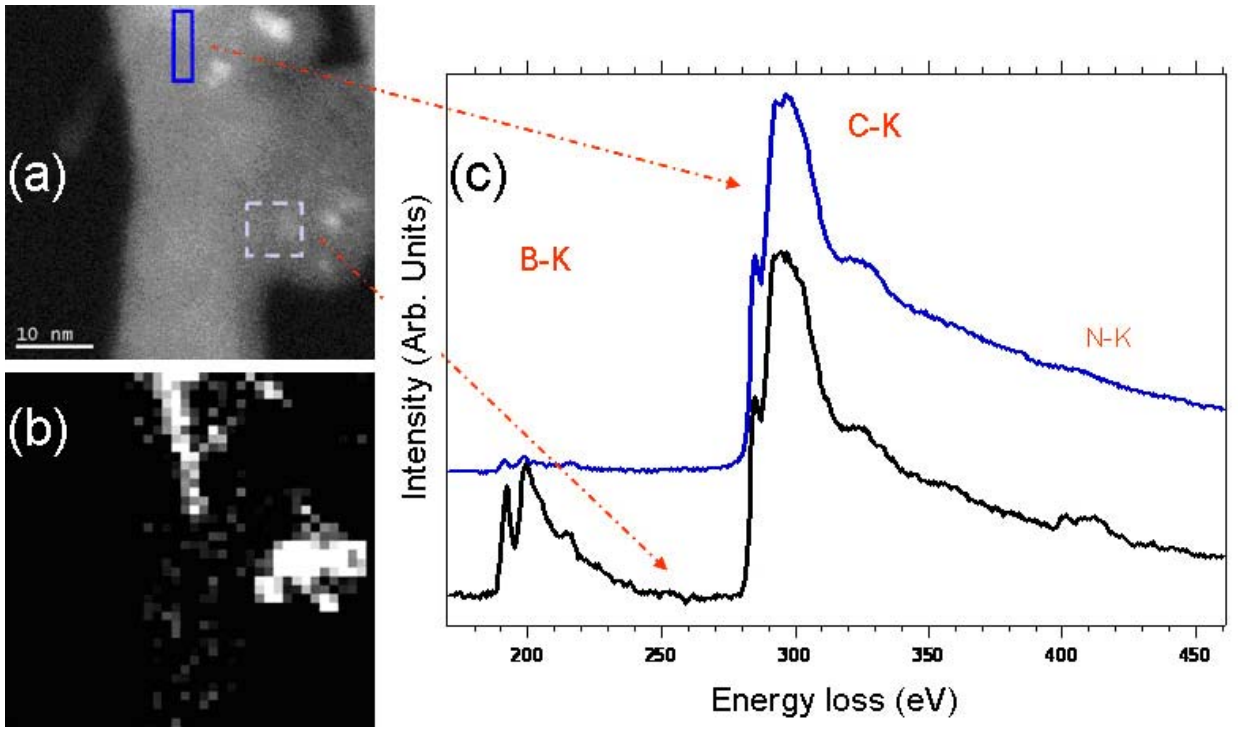

Figure 2. (a) HAADF image of a bundle of NTs containing also some nanoparticles embedded in this rope structure. In this area, a 40x40 SPIM have been recorded. (b) Boron elemental map extracted from this SPIM. (c) EEL spectra obtained form the marked areas of Fig. 2 (b). 